\title{
Magnetoresistance Effect of Thin Films Made of Single Graphite Crystals
}

\author{
Yoshiko Ohashi*, Tatsuya Hironaka**, Toshiharu Kubo* and Kazuo Shiiki*
}

(Received August 1, 2000, Accepted November 20, 2000)

\begin{abstract}
The magnetic field dependence of the transverse magnetoresistance $\Delta \rho / \rho_{0}$ and the Hall coefficient $R_{\mathrm{H}}$ was measured for the graphite films of various thickness ranging from around 20 to $110 \mathrm{~nm}$ at temperatures between 4.2 $\mathrm{K}$ and $300 \mathrm{~K}$. High quality of the crystallinity of these graphite films prepared by cleaving could be confirmed from the observation of Shubnikov-de Haas oscillation in $R_{\mathrm{H}}$. The saturation tendency revealed in the resistivity $\rho$ versus magnetic field $B$ characteristics for graphite films thinner than about $50 \mathrm{~nm}$ in the strong magnetic field region at low temperature, while as for all graphite films in this study, $\rho$ varied in proportion to $B^{n}(n>1)$ in the strong magnetic field region at high temperature. According to the transport theory for the simple two carrier model in the strong magnetic field region, this saturation tendency should be due to the difference between electron and hole densities. It may be caused by some change of energy band structure in thin films. Furthermore, at high temperature, $\rho$ versus $B$ characteristics, common to all these graphite films, may be explained by the equal number density of electrons and holes due to thermal excitation.
\end{abstract}

\section{KEYWORDS : Graphite, Nano-scale thickness, Hall effect, Magnetoresistance, Temperature dependence}

\section{Introduction}

Since graphite crystal is easy to be cleaved because of its layer structure, we can expect to realize making very thin films, the thickness of which is nano-scale. Recently, we have actually accomplished to make thin films of thickness ranging from around $30 \mathrm{~nm}$ to $110 \mathrm{~nm}$, and have measured the temperature dependence of electrical resistivity from liquid helium temperature to room temperature' ${ }^{1)}$. The experimental results could be expressed by a simple two band model ${ }^{2)}$ and the Sugihara's theory for lattice vibration in thin-carbon films ${ }^{3)}$, and the overlap energy $E_{0}$ of conduction and valence bands for the thin graphite films were estimated. Consequently, it was found that as the film thickness decreased, the value of the band overlap energy $E_{0}$ decreased gradually, though the relaxation time calculated from the model mentioned above was too short.

In this study we measured the magnetic field dependence of the transverse magnetoresistance $\Delta \rho / \rho_{0}$ and the Hall coefficient $R_{\mathrm{H}}$ for the graphite films of various thickness ranging from 20 to $110 \mathrm{~nm}$ at high temperature, up to $300 \mathrm{~K}$ to obtain the more inclusive characteristics. Especially, we focused our attention on the magnetic field dependence of resistivity $\rho$ at various temperature and tried to explain its anomalous behavior for thin films at high field region at low temperature comparing with that at high temperature.

\section{Experimental}

All of the specimens measured in this study were made of the same bulk crystal of kish graphite $(\mathrm{KG})$ as that used in the previous work ${ }^{1)}$, the residual resistivity ratio $(r r r)$ value of which was 32.3 . The method of the sample preparation was also the same as that described in the previous work'1), though the films were not worked into bridge type specimen in order to avoid the damage to the sample as much as possible. Van der Pauw's method $^{4)}$ was used for the measurements of Hall coefficient and transverse magnetoresistance effect. Because this method is available to carry out these measurements of a flat sample of arbitrary shape. Accordingly, we could measure a little tiny sample, the area of which was smaller than those used in the previous work, though we had to use uniform part of the film. The magnetic field was applied perpendicular to the film surface at the various temperature between $4.2 \mathrm{~K}$ and $300 \mathrm{~K}$.

\section{Results and Discussion}

The Hall coefficient $R_{\mathrm{H}}$ versus magnetic field of graphite films at liquid helium temperature is shown in Fig.1 corresponding to the film thickness of $18,23,35$ and $45 \mathrm{~nm}$ respectively. Shubnikov de-Haas oscillation can be observed for all of these specimens even for the thinnest one. This observation means that these graphite films should have high quality of the crystallinity. Therefore, the thinner films will not contain more defects by cleaving. Moreover, it was found that the period of Shubnikov-de Haas oscillation in $R_{\mathrm{H}}$ is almost twice as long as that of bulk crystals ${ }^{5}$. It means that the cross section of Fermi surface in thin films is about half area of that in bulk crystals and the carrier density is also about half as much as that in bulk crystals ${ }^{5}$. It is consistent with the previous work ${ }^{1)}$, qualitatively. As shown in Fig.2, the dependence of the transverse magnetoresistance $\Delta \rho / \rho_{0}$ of graphite films on magnetic field B is much different from that of bulk graphite at liquid helium temperature. Supposing the common relationship, $\Delta \rho / \rho_{0} \propto B^{n}$ corresponding to each specimen of graphite films, the exponent $\mathrm{n}$ seems to be smaller than unity in strong magnetic field region. Namely, it suggests the saturation tendency of $\Delta \rho / \rho_{0}$, as the

* Faculty of Science and Technology, Keio University : 3-14-1 Hiyoshi, Kohoku-ku, Yokohama, 223-8522, Japan

** Northern Telecom Japan Inc. : 3-19-23 Minamiazabu, Minato-ku, Tokyo 106-0047, Japan 


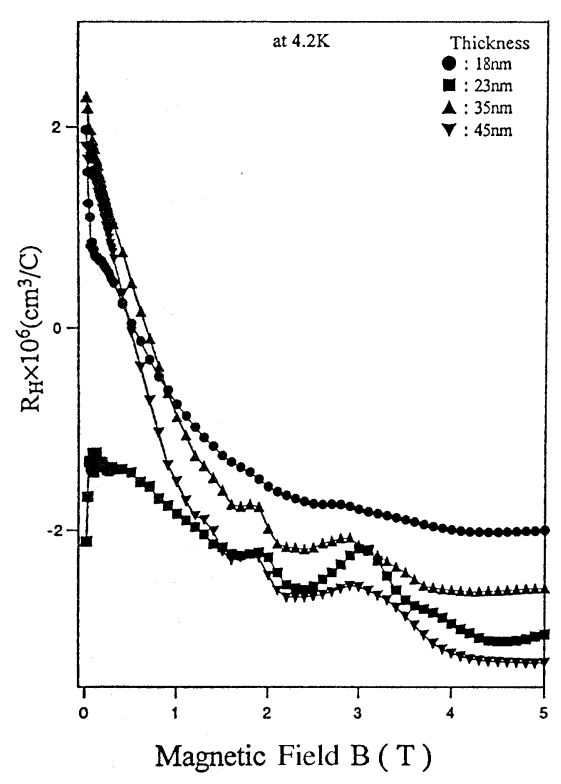

Fig.1 Hall coefficient $R_{\mathrm{H}}$ of thin graphite crystals as a function of magnetic field $B$ at $4.2 \mathrm{~K}$. The sample thickness is 18 , 23,35 , and $45 \mathrm{~nm}$, respectively.

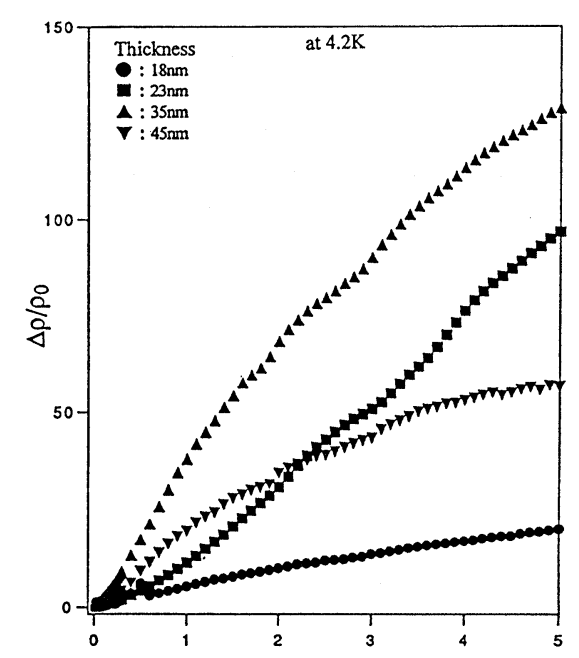

Magnetic Field B ( T )

Fig.2 Transverse magnetoresistance effect $\Delta \rho / \rho_{0}$ of thin graphite crystals as a function of magnetic field $B$ at $4.2 \mathrm{~K}$. The sample thickness is $18,23,35$, and $45 \mathrm{~nm}$, respectively.

magnetic field increases. In order to obtain more detailed characteristics, the electric resistivity for thicker films was measured as a function of magnetic field $B$ at higher temperatures additionally as shown in Fig.3. It should be noted that the magnetic field dependence of the resistivity $\rho$ is fundamentally equivalent to that of magnetoresistance effect $\Delta \rho / \rho_{0}$. The

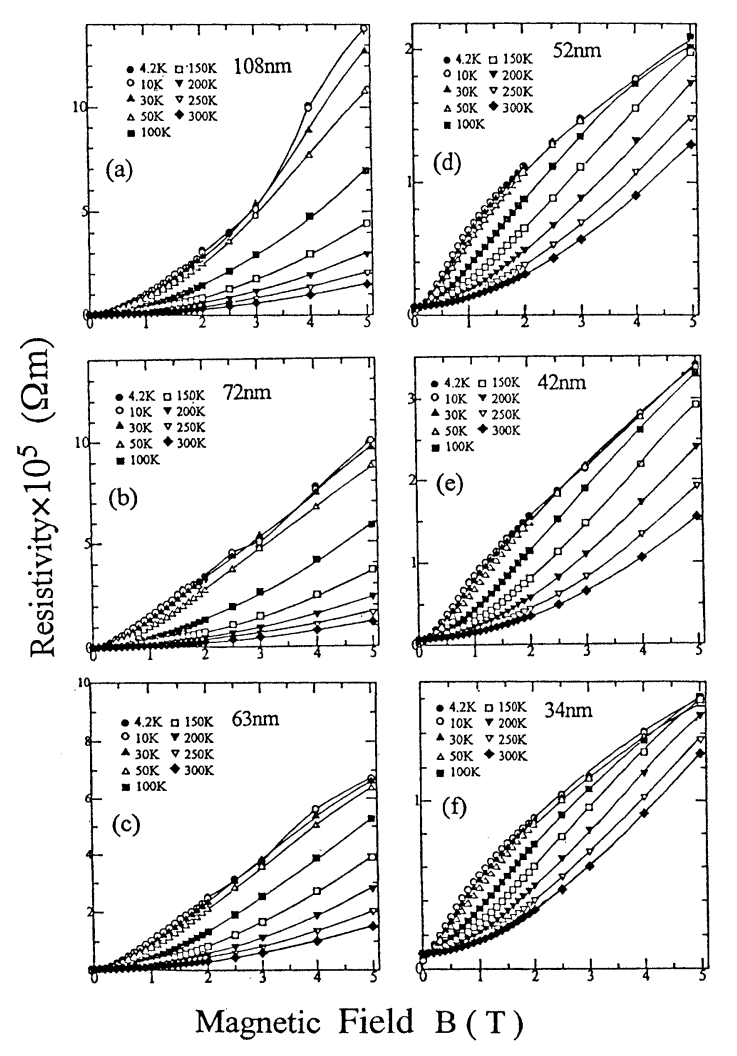

Fig.3 The resistivity as a function of magnetic field $B$ at various temperature. The thickness :
(a) $108 \mathrm{~nm}$, (b) $72 \mathrm{~nm}$, (c) $63 \mathrm{~nm}$, (d) $52 \mathrm{~nm}$,
(e) $42 \mathrm{~nm}$, (f) $34 \mathrm{~nm}$.

thickness of the specimens is ranging from around $30 \mathrm{~nm}$ to 110 $\mathrm{nm}$, and temperature was changed from 4.2 to $300 \mathrm{~K}$. Since the temperature increases, the magnetic field dependence of the resistivity for each specimen genelally weakens. As shown in Fig.3 (a), Fig.3 (b) and Fig.3 (c), n value of the graphite films thicker than about $50 \mathrm{~nm}$ is always larger than unity at all temperatures within this experiment. Because, the curvature of all these characteristics is upward. On the other hand, as shown in Fig.3 (d), Fig.3 (e) and Fig.3 (f), n value of the graphite films thinner than about $50 \mathrm{~nm}$ is smaller than unity at low temperature, because of the downward curvature, while their $n$ value becomes larger than unity at high temperature. At the highest temperature of $300 \mathrm{~K}$, value $\mathrm{n}$ of all specimens is larger than unity even in the thinnest film, that is similar to that of bulk graphite crystals ${ }^{6)-8)}$, except for the precise value of $n$. The effect of temperature on magnetic field dependence of the electric resistivity is more remarkable in Fig.4 (a) and Fig.4 (b), corresponding to the lowest temperature $4.2 \mathrm{~K}$, and the highest temperature $300 \mathrm{~K}$, respectively. At liquid helium temperature, the saturation tendency can be found for the films thinner than about $50 \mathrm{~nm}$, while the saturation tendency can not be clearly recognized for the films thicker than about $50 \mathrm{~nm}$, though the Shubnikov-de Haas effect is superimposed as shown in Fig.4 (a). 

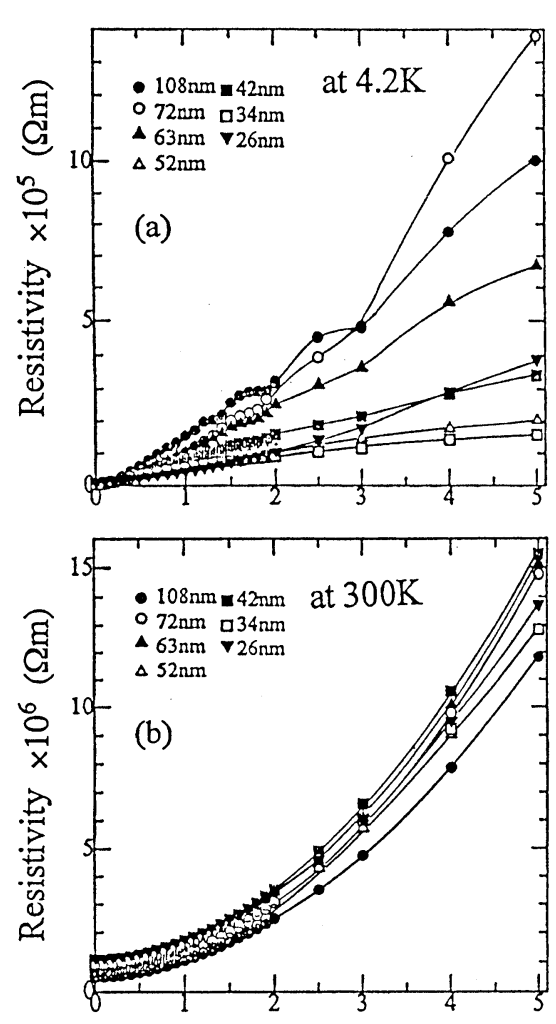

Magnetic Field B ( T )

Fig.4 The resistivity as a function of magnetic field $B$ for various thickness, at both low and high temperatures for comparison. The temperature :

(a) $4.2 \mathrm{~K}$, (b) $300 \mathrm{~K}$.

On the other hand, the value $\mathrm{n}$ is larger than unity for all specimens at $300 \mathrm{~K}$, and there seems neither saturation nor remarkable thickness dependence on resistivity versus magnetic field characteristics as shown in Fig.4 (b). The effect of the lattice vibration should be predominant at room temperature.

Now, applying the well known classical equation of motion for charged particles in electromagnetic field to the system composed of two kinds of carriers*, these anomalous behaviors will be explained. When the strong magnetic field $\boldsymbol{B}(0,0, B)$ is applied perpendicular to the film surface corresponding to $x$ $y$ plane, $x$ component of current density $j_{x}$ in the Hall configuration under the condition of strong field approximation is expressed as follows :

$$
j_{x}=\left(\frac{n_{\mathrm{e}} q_{\mathrm{e}}}{\mu_{\mathrm{c}}}+\frac{n_{\mathrm{h}} q_{\mathrm{h}}}{\mu_{\mathrm{h}}}\right) \cdot\left(\frac{1}{B}\right)^{2} E_{x}+\frac{\left(n_{\mathrm{e}} q_{\mathrm{c}}+n_{\mathrm{h}} q_{\mathrm{h}}\right)^{2}}{\left(\frac{n_{\mathrm{e}} q_{\mathrm{c}}}{\mu_{\mathrm{e}}}+\frac{n_{\mathrm{h}} q_{\mathrm{h}}}{\mu_{\mathrm{h}}}\right)} E_{x} \ldots \text { (1) }
$$

where $E_{x}$ : electric field, $\mu$ : mobility, $n$ : carrier density, $q$ : carrier charge. In this equation, $\mu, n$ and $q$ consist of two carriers corresponding to electrons and holes, each of them is indicated by $e$ and $h$, respectively. This equation indicates the magnetic field dependence of resistivity in the strong field region. If $n_{\mathrm{c}}=$ $n_{\mathrm{h}}$, the second term vanishes, then resistivity increases as the field increases. However, if $n_{\mathrm{c}} \neq n_{\mathrm{h}}$, as the magnetic field increases, the first term becomes smaller than the second term, drastically. Consequently, saturation appears in the resistivity versus magnetic field characteristics.

The reason why the saturation tendency appears is that the carrier density of thin graphite films is less than that of bulk crystals, because of the lower overlap energy between conduction and valence bands'), and as the result, the difference between densities of electrons and holes, $n_{\mathrm{h}}-n_{\mathrm{e}}$ should be effective to the resistivity in the strong field at low temperature. On the other hand, many electrons and holes excited as carriers at high temperature, and then, the difference between densities of electrons and holes becomes negligible small in comparison with total carrier density. Therefore, the resistivity varies in proportion to $B^{\mathrm{n}}(\mathrm{n}>1)$ in the strong magnetic field, that is similar to the case of the bivalent metal. Moreover, as the mobility becomes very small at high temperature, the condition of strong field can not be satisfied. In these considerations, we assumed that the mobility of both electrons and holes was independent of wave vector $\boldsymbol{k}$, however we should make more precise explanation in the future.

\section{Summary}

Magnetic field dependence of $R_{\mathrm{H}}$ and of $\Delta \rho / \rho_{0}$ in the graphite films were measured for various thickness ranging from around 30 to $110 \mathrm{~nm}$ at temperatures between $4.2 \mathrm{~K}$ and $300 \mathrm{~K}$. High quality of the crystallinity of the graphite films prepared by cleaving could be confirmed from the observation of Shubnikovde Haas oscillation in $R_{\mathrm{H}}$. In the strong magnetic field region, the resistivity versus magnetic field characteristics revealed saturation tendency in thin graphite films at low temperature, while the resistivity varied with the magnetic field in proportion to $B^{\text {n }}(\mathrm{n}>1)$ for every films at high temperature and for the thick films at low temperature. These experimental results of the resistivity versus magnetic field characteristics were explained by applying the simple classical transport theory to graphite films. That is, the difference between densities of electrons and holes is predominant due to the low overlap energy between conduction and valence bands in thin graphite films at low temperature. On the other hand, at high temperature and for the thick films, equal number of electrons and holes due to thermal excitation should play an important role, which is similar to the case of the bivalent metal; otherwise the condition of strong magnetic field could not be satisfied owing to the very small mobility.

\section{Acknowledgments}

The authors wish to thank Professor Hishiyama of Musashi Institute of Technology for kindly supplying a precious KG crystal with the $r r$ value of 32.3 and to thank Professor Yasuda of Tokyo Institute of Technology for his valuable support. A part of this work was supported by Keio Gijuku Academic Development Funds.

* Note : Eq (1) is derived from the equation of motion for charged particle in electromagnetic field as follows : 


$$
m \frac{\mathrm{d} \boldsymbol{v}}{\mathrm{d} t}+\frac{m}{\tau} \boldsymbol{v}=q(\boldsymbol{E}+[\boldsymbol{v} \times \boldsymbol{B}]),
$$

where $m:$ mass, $\boldsymbol{v}:$ velocity, $\tau$ : relaxation time, $q$ : charge, $\boldsymbol{E}$ : electric field and $\boldsymbol{B}$ : magnetic field. $\boldsymbol{v}, \boldsymbol{E}$ and $\boldsymbol{B}$ are composed of $x, y$, and $z$ components, respectively.

When the magnetic field $\boldsymbol{B}(0,0, \boldsymbol{B})$ is applied perpendicular to the film surface corresponding to $x$ - $y$ plane, the current densities $j_{\mathrm{x}}$ and $j_{\mathrm{y}}$ in the Hall configuration, are expressed as

$$
j_{\mathrm{x}}=n q v_{\mathrm{x}}, j_{\mathrm{y}}=n q v_{\mathrm{y}}
$$

where $n$ is carrier density. Using cyclotron frequency $\omega_{\mathrm{c}}=\frac{q B}{m^{*}}$, mobility $\mu=\frac{q \tau}{m^{*}}$, and $\omega_{\mathrm{c}} \tau=\mu B$, then velocity component $v_{\mathrm{x}}$ and $v_{\text {y }}$ are obtained as follows :

$$
v_{\mathrm{x}}=\frac{\mu}{1+\left(\omega_{\mathrm{c}} \tau\right)^{2}}\left(E_{\mathrm{x}}+\omega_{\mathrm{c}} \tau E_{\mathrm{y}}\right), v_{\mathrm{y}}=\frac{\mu}{1+\left(\omega_{\mathrm{c}} \tau\right)^{2}}\left(E_{\mathrm{y}}-\omega_{\mathrm{c}} \tau E_{\mathrm{y}}\right)
$$

In the strong magnetic field region, where $\omega_{\mathrm{c}} \tau \gg 1$,

$$
\begin{aligned}
& v_{\mathrm{x}} \approx \frac{\mu}{\left(\omega_{\mathrm{c}} \tau\right)^{2}}\left(E_{\mathrm{x}}+\omega_{\mathrm{c}} \tau E_{\mathrm{y}}\right)=\frac{1}{\mu B^{2}}\left(E_{\mathrm{x}}+\mu B \cdot E_{\mathrm{y}}\right), \cdots(\mathrm{N} 4 \mathrm{a}) \\
& v_{\mathrm{y}} \approx \frac{\mu}{\left(\omega_{\mathrm{c}} \tau\right)^{2}}\left(E_{\mathrm{y}}-\omega_{\mathrm{c}} \tau E_{\mathrm{x}}\right)=\frac{1}{\mu B^{2}}\left(E_{\mathrm{y}}-\mu B \cdot E_{\mathrm{x}}\right) \cdots(\mathrm{N} 4 \mathrm{~b})
\end{aligned}
$$

Here, $v_{\mathrm{x}}$ and $v_{\mathrm{y}}$ consist of two terms corresponding to electrons and holes, each of them is indicated by e and $h$, respectively. Then $j_{\mathrm{y}}$ is rewritten as follows :

$$
\begin{aligned}
j_{\mathrm{y}} & =n_{\mathrm{e}} q_{\mathrm{e}} v_{\mathrm{ey}}+n_{\mathrm{h}} q_{\mathrm{h}} v_{\mathrm{hy}} \\
& =\frac{n_{\mathrm{c}} q_{\mathrm{c}}}{\mu_{\mathrm{c}} \cdot B^{2}}\left(E_{\mathrm{y}}-\mu_{\mathrm{c}} B \cdot E_{\mathrm{x}}\right)+\frac{n_{\mathrm{h}} q_{\mathrm{h}}}{\mu_{\mathrm{h}} \cdot B^{2}}\left(E_{\mathrm{y}}-\mu_{\mathrm{h}} B \cdot E_{\mathrm{x}}\right) .
\end{aligned}
$$

In the case of Hall configuration, $j_{y}=0$.

Then, eqs. (N6), (N7) are obtained.

$$
\begin{aligned}
& \left(\frac{n_{\mathrm{c}} q_{\mathrm{c}}}{\mu_{\mathrm{c}}}+\frac{n_{\mathrm{h}} q_{\mathrm{h}}}{\mu_{\mathrm{h}}}\right) E_{\mathrm{y}}=B\left(n_{\mathrm{c}} q_{\mathrm{c}}+n_{\mathrm{h}} q_{\mathrm{h}}\right) E_{\mathrm{x}}=0 \\
& E_{\mathrm{y}}=\frac{n_{\mathrm{c}} q_{\mathrm{c}}+n_{\mathrm{h}} q_{\mathrm{h}}}{\frac{n_{\mathrm{c}} q_{\mathrm{c}}}{\mu_{\mathrm{c}}}+\frac{n_{\mathrm{h}} q_{\mathrm{h}}}{\mu_{\mathrm{h}}}} B \cdot E_{\mathrm{x}}
\end{aligned}
$$

Using above equations, $x$ component of current density $j_{\times}$can be derived as follows :

$$
\begin{aligned}
j_{\mathrm{x}} & =n_{\mathrm{c}} q_{\mathrm{c}} v_{\mathrm{cx}}+n_{\mathrm{h}} q_{\mathrm{h}} v_{\mathrm{hx}} \\
& =\frac{1}{B^{2}}\left\{\frac{n_{\mathrm{c}} q_{\mathrm{c}}}{\mu_{\mathrm{c}}}\left(E_{\mathrm{x}}+\mu_{\mathrm{c}} B \cdot E_{\mathrm{y}}\right)+\frac{n_{\mathrm{h}} q_{\mathrm{h}}}{\mu_{\mathrm{h}}}\left(E_{\mathrm{x}}+\mu_{\mathrm{h}} B \cdot E_{\mathrm{y}}\right)\right\} \\
& =\left(\frac{n_{\mathrm{c}} q_{\mathrm{c}}}{\mu_{\mathrm{c}}}+\frac{n_{\mathrm{h}} q_{\mathrm{h}}}{\mu_{\mathrm{h}}}\right)\left(\frac{1}{B}\right)^{2} E_{\mathrm{x}}+\frac{\left(n_{\mathrm{c}} q_{\mathrm{c}}+n_{\mathrm{h}} q_{\mathrm{h}}\right)^{2}}{\left(\frac{n_{\mathrm{c}} q_{\mathrm{c}}}{\mu_{\mathrm{c}}}+\frac{n_{\mathrm{h}} q_{\mathrm{h}}}{\mu_{\mathrm{h}}}\right)} E_{\mathrm{x}}
\end{aligned}
$$

\section{Reference}

1) Y. Ohashi, K. Koizumi, T. Yoshikawa, T. Hironaka and K. Shiiki, TANSO 1997 [No.180] 235-238.

2) C. A. Klein, J. Appl. Phys. 35 (1964) 2947-2957.

3) K. Sugihara, Phys. Rev. B37 (1988) 7063-7069.

4) L. J. van der Pauw, Philips Research Reports 13 (1958) $1-9$.

5) to be published.

6) D. E. Soule, Phys. Rev. 112 (1958) 698-707.

7) Y. Kaburagi, J. Phys. C: Solid State Phys. 15 (1982) 54255440.

8) Y. Kaburagi and Y. Hishiyama, Carbon 33 (1995) 15051506. 